\title{
El manejo emocional en docentes granadinos en relación a la satisfacción en el trabajo
}

Aida M. Centeno Barrera, Alicia Herrera Ojea, Govinda L. López Vidaurre, Carmen N. Machado Castilla y Laura Trujillo Patón. Universidad de Granada

\author{
Recepción: 20 de mayo de 2016 | Revisión: 21 de mayo de 2016 | Aceptado: 30 de mayo de 2016 \\ Correspondencia: aidacenba@correo.ugr.es \\ Citar: Centeno, A., Herrera, A., López, G., Machado, C. y Trujillo, L. (2016). El manejo emocional en docentes granadinos en \\ relación a la satisfacción en el trabajo. ReiDoCrea, 5(2), 14-20.
}

\begin{abstract}
Resumen: El propósito de este estudio descriptivo, que ha contado con 40 sujetos, es conocer la relación entre las diferentes dimensiones de la satisfacción en el trabajo y la inteligencia emocional en una muestra de profesores de dos Institutos de Educación Secundaria de Granada. Se ha realizado utilizando el Cuestionario de Satisfacción Laboral S20/23 y el TMMS-24 que mide la inteligencia emocional. Tras un análisis de los datos obtenidos, se ha podido comprobar que no existía correlación alguna entre nuestras variables y, por otro lado, que no había diferencias significativas. Es por esto que no se da evidencia a favor de la literatura anterior y, con ello, se aportan cuáles son las limitaciones del presente estudio para intentar ver cuál es el motivo de estos resultados.
\end{abstract}

Palabras clave: Inteligencia emocional | Satisfacción en el trabajo

Emotional Control in Teachers from Granada in Relation to Job Satisfaction

Abstract: The purpose of this descriptive study, in which 40 subjects participated, is to determine the relationship between different aspects of job satisfaction and emotional intelligence in a sample of Secondary School teachers from Granada. Two questionnaires were used including the Job Satisfaction Questionnaire S20/23 and the TMMS-24. The data analysis shows that there was no correlation between these variables and that there were no significant differences. No evidence was found supporting the earlier literature, hence the inclusion of the limitations of the present study in an attempt to determine the underlying cause of the outcome.

Key words: Emotional intelligence | Job satisfaction

\section{Introducción}

En el panorama actual, la inteligencia emocional se ha convertido en uno de los temas de mayor interés dentro de la comunidad científica social y en el ámbito público (BarOn, 2006). Apareció revolucionando la concepción inicial de inteligencia asumida en psicología, las relaciones sociales humanas y el ámbito laboral en general, entre otras muchas áreas. Pero, ¿cómo definimos exactamente la inteligencia emocional (IE)? Mayer y Salovey (1997), la definieron en su modelo como "la habilidad de percibir y expresar correctamente las emociones, utilizando dicha información para razonar y comprender el mundo que nos rodea y, de este modo, poder regularlas". Dentro de la inteligencia emocional estos autores destacan cuatro habilidades: la de percibir, valorar y expresar emociones; la de comprender las emociones; la de tener en cuenta los sentimientos cuando razonamos o solucionamos un problema $\mathrm{y}$, por último, la habilidad de saber regularlas.

Un número considerable de investigaciones ha demostrado que esta variable guarda una estrecha relación con la satisfacción laboral (Hongbiao, 2015; O’Boyle, Humphrey, Pollack, Hawver y Story, 2011; Pena y Extremera, 2012; Shi, Yan, Xuqun y Li, 2014.). Peiró (1991) define la satisfacción laboral como "el conjunto de actitudes que una persona tiene hacia su trabajo, entendiendo por actitud aquella configuración de placer o estado emocional positivo resultante de una o varias experiencias de trabajo". En lo que respecta a dicha relación, Palomera, Gil-Olarte y Brackett (2006) señalan que en el contexto educativo la IE es uno de los factores individuales que permiten explicar la resistencia de los profesores ante el estrés y por tanto llegar a ser uno de los factores 
preventivos del malestar docente. Estas habilidades se ponen en práctica tanto con el alumnado como con los padres, así como con los compañeros de trabajo, mejorando de esta forma el bienestar laboral. Anani (2011) reitera que una IE alta es muy importante para los docentes, ya que refleja las habilidades sociales que los ayudan en las interacciones con los estudiantes. De este modo, tienen éxito como profesionales, desarrollándose y progresando en sus instituciones, mientras que los alumnos mejoran como estudiantes.

A la luz de los hallazgos previos, y ya que la mayoría de investigación se ha realizado en el campo de la enseñanza (Ganzach, 1998; Hongbiao, 2015; Pena y Extremera, 2012), se propone como objetivo para el presente trabajo estudiar este efecto en el profesorado de la Enseñanza Secundaria. Partiendo de la idea de que la IE ayuda a las personas a percibir y expresar correctamente nuestras emociones, y a comprender el mundo que nos rodea (Mayer y Salovey, 1997), se espera que los docentes con niveles más altos experimenten menos consecuencias negativas de estrés y vean satisfechas sus expectativas y metas laborales, es decir, muestren una mayor satisfacción laboral.

\section{Método}

\section{Participantes}

En esta investigación participaron 27 mujeres y 16 hombres (40 participantes en total) con edades comprendidas entre 27 y 59 años $(M=44,20 ; D T=8,76)$, que en la actualidad ejercen de profesores en dos Institutos de Secundaria, en concreto el Instituto Juan XXIII de Granada y al Instituto Acrebite de Baza, en la misma provincia. Tenían entre 1 y 40 años de antigüedad laboral $(M=15,17 ; D T=9,97)$. Todos ellos tenían el castellano como lengua materna y su natalidad era española; el nivel socioeconómico y cultural de cada uno de ellos era medio. La participación de los trabajadores en este estudio fue voluntaria y anónima.

\section{Diseño}

Se realizó un estudio descriptivo transversal que intenta describir la relación entre satisfacción laboral e inteligencia emocional en trabajadores de dos Institutos de secundaria de Granada.

\section{Procedimiento}

Se contactó con el orientador de cada instituto, quien se encargó de entregar y recoger los cuestionarios a cada profesor, ya que no se permitió que los cuestionarios fueran aplicados por personas externas al centro. Los profesores rellenaron el cuestionario en el lugar de trabajo o fuera de él, según estimasen oportuno. El orientador fue informado de las instrucciones que debía proporcionar a cada profesor y de cómo debía resolver cualquier duda. Todos los participantes recibieron los cuestionarios junto con una hoja de consentimiento informado. Se les dieron las siguientes instrucciones por escrito: "En el interior de este cuadernillo encontrará una serie de afirmaciones destinadas a conocer distintos aspectos de su vida emocional y labora. Le rogamos que lea detenidamente las instrucciones y pregunta cualquier duda que surja. 1. Responda con sinceridad, los cuestionarios son anónimos. 2. No medite sus respuestas. Dé la primera que se le venga a la mente de modo natural. 3. Algunas cuestiones pueden no ser aplicables a su situación; no obstante, intente elegir la que mejor se adapte a sus circunstancias. 4. Asegúrese de no dejar ninguna pregunta en blanco y de contestar todas en el orden en que aparecen. No pase a la siguiente 
página sin haber acabado la anterior. 5. Debe saber que la información recogida no será utilizada con fines distintos a los indicados."

\section{Instrumentos}

Hemos utilizado 2 tipos de cuestionarios en este estudio:

1. TMMS-24 (Trait-Meta Mood Scale, Salovey y Mayer, 1995; adaptado al castellano por Fernández-Berrocal et al, 1998, 2002. Uso de versión reducida). Está basado en el Trait-Meta Mood Scale (TMMS) de Salovey y Mayer. Consta de 24 ítems formados por enunciados verbales cortos en los que se evalúa la inteligencia emocional en una escala Likert con cinco opciones de distinto nivel de conformidad con ese ítem, que van desde (1) Nada de acuerdo a Totalmente de acuerdo (5). Está compuesto por tres dimensiones de la Inteligencia Emocional con 8 ítems cada una de ellas. Estas dimensiones son Percepción Emocional ("Presto mucha atención a los sentimientos"), Comprensión de Sentimientos ("Puedo llegar a comprender mis sentimientos") y Regulación Emocional ("Aunque me sienta mal, procuro pensar en cosas agradables"). El alfa de Cronbach para la primera es 0.90 ; en el caso de la segunda es 0.90 ; y la tercera es 0.86 .

2. Cuestionario de Satisfacción Laboral S20/23 (Meliá y Peiró, 1989). Consta de 23 ítems formados por enunciados verbales cortos en los que se evalúa la satisfacción laboral en una escala Likert con siete opciones de distinto nivel de conformidad, que van desde (1) Muy insatisfecho a Muy satisfecho (7). Tiene una estructura de cinco secciones: Satisfacción con la supervisión, con 6 ítems ("Las relaciones con sus superiores"); Satisfacción con el ambiente físico, con 5 ítems ("La limpieza, higiene y salubridad de su lugar de trabajo"); Satisfacción con las prestaciones recibidas, con 5 ítems ("El salario que usted recibe"); Satisfacción intrínseca del trabajo, con 4 ítems ("Las satisfacciones que le produce su trabajo por sí mismo"); Satisfacción con la participación, con 3 ítems ("Su participación en las decisiones de su departamento o sección").

El alfa de Cronbach para la primera dimensión tiene un valor de 0.95 ; la satisfacción con el ambiente físico tiene un alfa de 0.87; para la tercera dimensión alcanza un valor de 0.79 ; la satisfacción intrínseca presenta un valor de 0.96; y finalmente la satisfacción con la participación tiene un alfa de 0.88. La consistencia interna para la escala total de Satisfacción Laboral es de 0.96.

Además se recogieron los siguientes datos socio-demográficos de cada participante: edad, sexo, puesto de trabajo, categoría laboral, nivel de estudios, situación laboral, horario laboral, cantidad de horas trabajadas semanalmente y antigüedad en la empresa.

\section{Resultados}

Los datos han sido analizados con el programa estadístico SPSS 20.0. Los análisis realizados han sido descriptivos, correlaciones de Pearson y pruebas T student para muestras independientes. Para poder recabar información sobre nuestras hipótesis, decidimos realizar el procedimiento estadístico de correlaciones de Pearson entre nuestras variables: Inteligencia emocional (IE) y Satisfacción laboral (SL).

Con la finalidad de obtener unos mayores resultados comparamos las dimensiones de cada variable. No hemos encontrado correlaciones entre nuestras variables (Tabla 1), ni tampoco entre sus dimensiones. Obtuvimos que la satisfacción laboral total no tenía correlación con ninguna de las dimensiones de inteligencia emocional. 
Tabla 1. Correlación Inteligencia emocional y Satisfacción emocional

Supervisión

Ambiente físico

Prestaciones

Intrínseca

Participación

Satisfacción laboral

. La correlación es significativa a nivel 0.05 (bilateral).

**. La correlación es significativa a nivel 0.01 (bilateral).

Debido a no haber encontrado la relación esperada entre nuestras variables, pasamos a ver si había diferencias significativas entre distintos grupos que realizamos en este estudio. En un primer momento, realizamos una prueba $T$ para comprobar si había diferencias entre hombres y mujeres en cuanto a satisfacción laboral e inteligencia emocional. De este modo, comprobamos que no hay diferencias significativas entre ambos sexos en ninguna de las dimensiones de nuestras variables: inteligencia emocional y satisfacción laboral como podemos comprobar en la Figura 1.

Figura 1. Diferencias entre sexos

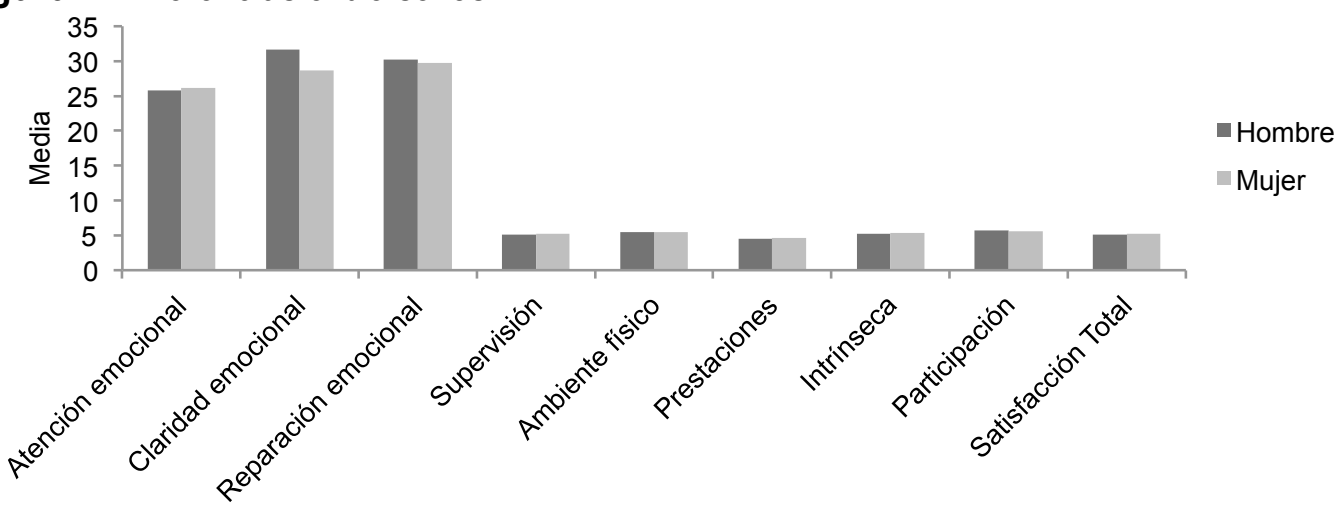

Igualmente procedimos a realizar este mismo análisis pero diferenciando entre el número de años trabajados. Dividimos la muestra en dos grupos: más de 15 años trabajados y menos de 15 años trabajados debido a que esta era la media que se encontró en cuanto a esta variable. Tampoco fueron halladas diferencias significativas entre estos grupos como se puede comprobar en la Figura 2.

Figura 2. Diferencias en años trabajados

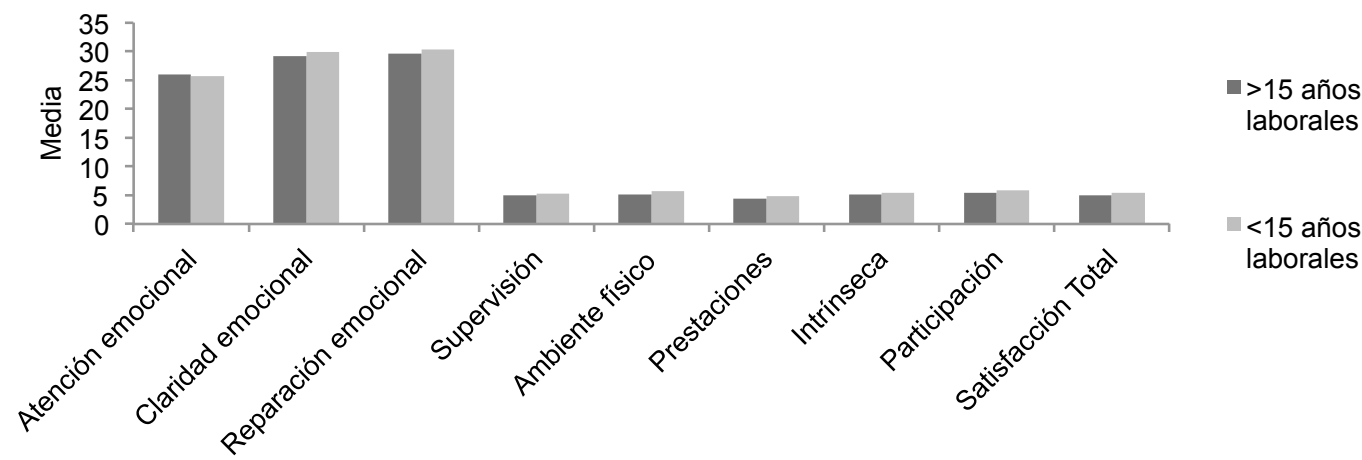


Por otra parte, y dejando de la lado nuestras hipótesis un momento, decidimos comprobar qué correlación había entre las distintas dimensiones de nuestros constructos y así conseguíamos comprobar la validez de los instrumentos usados.

En el TMMS-24, comprobamos que se daba una correlación positiva y significativa entre todas las dimensiones exceptuando reparación emocional y atención emocional (Tabla 2).

Tabla 2. Correlación entre las dimensiones de IE

\begin{tabular}{lccc|}
\hline & Atención emocional & Claridad emocional & Reparación emocional \\
\cline { 2 - 4 } Atención emocional & -- & $.399^{*}$ & .099 \\
Claridad emocional & $.399^{*}$ & -- & $.431^{* *}$ \\
Reparación emocional & .099 & $.431^{* *}$ & -- \\
\hline${ }^{*}$. La correlación es significativa a nivel 0.05 (bilateral). & & \\
${ }^{* *}$. La correlación es significativa a nivel 0.01 (bilateral). & & \\
\hline
\end{tabular}

Por otro lado en el Cuestionario de Satisfacción Laboral S20/23, pudimos comprobar que las correlaciones que se daban entre las distintas dimensiones eran positivas y altas entre todas ellas (Tabla 3). Por tanto, destacamos la validez de este cuestionario en el que encontramos correlaciones muy altas como puede ser entre "supervisión" y "prestaciones" ( $r=.689 ; p<.01)$.

\begin{tabular}{|c|c|c|c|c|c|}
\hline & Supervisión & Ambiente físico & Prestaciones & Intrínseca & Participación \\
\hline Supervisión & - & $.439^{* *}$ & $689^{* *}$ & $.456^{* *}$ & $.760^{* *}$ \\
\hline Ambiente físico & $.439^{* *}$ & - & $.384^{*}$ & $.375^{\star}$ & $.409^{* *}$ \\
\hline Prestaciones & $.689^{* *}$ & $.384^{*}$ & - & $.614^{* *}$ & $.568^{* *}$ \\
\hline Intrínseca & $.456^{* *}$ & $.375^{\star}$ & $.614^{* *}$ & -- & $.401^{*}$ \\
\hline Participación & $.760^{* *}$ & $.409^{* *}$ & $.568^{* *}$ & $.401^{*}$ & -- \\
\hline
\end{tabular}

\section{Discusión}

Tras analizar nuestros datos podemos llegar a varias conclusiones sobre nuestra muestra. Por un lado, no vemos cumplida nuestra hipótesis inicial ya que no conseguimos hallar una correlación entre la satisfacción laboral y la inteligencia emocional ni entre las diferentes dimensiones que las componen. Estos resultados han sido evidenciados también en otros estudios similares que no han podido demostrar esta relación entre inteligencia emocional y satisfacción laboral (Guadalupe y Tania, 2010; El Badawy y Magdy, 2015; Guy y Lee, 2015). Por otro lado, tampoco encontramos diferencias entre grupos de hombres y mujeres o entre distintos grupos de número años trabajados; la inexistencia de diferencias en estas variables entre géneros también se ha visto en estudios como el de El Badawy y Magdy, 2015.

Estos resultados pueden ser debidos a varios motivos. Procedemos ahora a detallároslo de una manera clara para aportar una mejor visión de conjunto a nuestro lector sobre la problemática con la que estamos lidiando.

Muy importante a destacar, pueden ser las influencias debidas a que la muestra seleccionada de docentes, a pesar de ser de colegios dispares, parece mostrar toda ella una alta satisfacción laboral, lo que nos impide hacer una comparación entre grupos con diferentes niveles de satisfacción laboral y limita las posibles correlaciones.

Quizá en estudios posteriores sería recomendable ampliar la muestra, pues solo con 40 sujetos algunos fenómenos pueden no verse claramente e, igualmente, no 
conseguirse una representación adecuada de la población. Otros estudios en esta línea usan muestras mucho mayores y consiguen demostrar la relación entre inteligencia emocional y satisfacción laboral (Shi, Yan y Xugun, 2014; Yin, 2015). También sería importante que la ampliación de la muestra estuviese repartida por centros escolares de características diversas como pueden ser la localización geográfica, el tipo de población en el que se encuentra o el nivel socioeconómico de los alumnos que acuden al centro.

Igualmente, debido al modo en el que fueron pasados los cuestionarios se puede haber visto afectada la deseabilidad social que mostraron los sujetos, pues el cuestionario era administrado por el orientador del centro, una persona conocida con la que trabajaban todos los días. Por esto pensamos que sería recomendable en futuras investigaciones en este campo, que se añadiese a los materiales un cuestionario que midiese la deseabilidad social pues la inteligencia emocional no es una variable que se vea libre de la influencia de este fenómeno (Fernández y Extremera, 2005).

Se ha demostrado que las puntuaciones en TMMS correlacionan de manera moderada con las variables de personalidad de los cinco grandes (Fernández y Extremera, 2005) por lo que añadir un cuestionario de personalidad podría aclararnos las puntuaciones que se obtengan en este cuestionario.

Todo lo dicho anteriormente, nos lleva a ver la importancia de este tipo de estudios en la actualidad. Los profesores juegan un gran papel en nuestro sistema educativo, por lo que no debemos ignorar su bienestar ni el reforzamiento de éste. Se ha comprobado que a más baja satisfacción laboral, mayor burnout (Hermosa, 2006). Durán, Extremera y Rey (2001) definen el burnout en el ámbito académico como: "Una respuesta de estado emocional negativo, generalmente acompañada por cambios fisiológicos potencialmente peligrosos, resultantes de aspectos del trabajo del profesor y mediados por la percepción de que las demandas del trabajo son amenazantes y por los mecanismos de afrontamiento que son activados para reducir esa amenaza" (Hermosa, 2006). Esta definición nos puede ayudar a vislumbrar la importancia de saber el estado emocional del profesorado en nuestro país.

Recomendamos por consiguiente proseguir esta línea de estudios, teniendo en cuenta todas las variables que pueden afectar a los resultados como hemos expuesto anteriormente. Así podremos llegar a datos que nos guíen en la creación de reformas o programas para paliar los problemas que podamos observar en el profesorado.

\section{Referencias}

Anani, N.N. (2011). Teachers: Emotional intelligence, job satisfaction, and organizational commitment. Journal of Workplace Learning, 24(4), 256-269.

Bar-On, R. (2006). The Bar-On model of emotional-social intelligence. Psicothema, 18, 13-25.

El Badawy, T.A., \& Magdy, M.M. (2015). Assessing the Impact of Emotional Intelligence on Job Satisfaction: An Empirical Study on Faculty Members with Respect to Gender and Age. International Business Research, 8(3), 67-78.

Fernandez, P. y Extremera, N. (2005). La Inteligencia Emocional y la educación de las emociones desde el Modelo de Mayer y Salovey. Revista Interuniversitaria de Formación del Profesorado, 19(3), 63-93.

Ganzach, Y. (1998). Intelligence and job satisfaction. Academy of Management Journal, 41(5), 526.

Guadalupe, C. y Tania, K. (2010). Inteligencia emocional y satisfacción laboral en docentes de educación inicial de la red $n^{\circ} 4$ de Ventanilla-Callao. Disertación Doctoral, Universidad San Ignacio de Loyola. 
Guy, M.E., \& Lee, H.J. (2015). How Emotional Intelligence Mediates Emotional Labor in Public Service Jobs. Review of Public Personnel Administration, 35(3), 261-277.

Hermosa, A. (2006). Satisfacción laboral y síndrome de "burnout" en profesores de educación primaria y secundaria. Revista Colombiana de Psicología, 15, 81-89.

Hongbiao, Y. (2015). The effect of teachers' emotional labour on teaching satisfaction: moderation of emotional intelligence. Teachers and Teaching, 21(7), 789-810.

Márquez, P.G.O., Brackett, M.A., y Martín, R.P. (2006). ¿Se perciben con inteligencia emocional los docentes?: posibles consecuencias sobre la calidad educativa. Revista de educación, 341, 687-704.

Mayer, J., \& Salovey, P. (1997). What is emotional intelligence? In Salovey P., \& Sluyter D. (1997). Emotional Development and Emotional Intelligence: Implications for Educators, 3-31. New York: Basic Book.

O'Boyle, E.H., Humphrey, R.H., Pollack, J.M., Hawver, T.H., \& Story, P.A. (2011). The relation between emotional intelligence and job performance: A meta-analysis. Journal of Organizational Behavior, 32, 788-818.

Palomera, R., Gil-Olarte, P. y Brackett, M. (2006). ¿Se perciben con inteligencia emocional los docentes? Posibles consecuencias sobre la calidad educativa. Revista de Educación, 341, 687-703.

Peiró J.M. (1991). Psicología de la Organización. Madrid: UNED.

Pena, M., \& Extremera N., (2012). Perceived Emotional Intelligence in Primary School Teachers and Its Relationship with Levels of Burnout and Engagement. Revista de Educación, 359, 604-627.

Shi, M., Yan X., Xuqun Y., \& Li J. (2014). Core Self-evaluations, Emotional Intelligence and Job Satisfaction in Chinese Soldiers. Social Indicators Research, 124, 221-229.

Yin, H. (2015). The effect of teachers' emotional labour on teaching satisfaction: moderation of emotional intelligence. Teachers and Teaching: Theory and Practice, 21(7), 789-810. 